\title{
Probing electron charging in nanocrystalline Si dots using Kelvin probe force microscopy
}

\author{
M. A. Salem, ${ }^{\text {a) }}$ H. Mizuta, and S. Oda ${ }^{\text {) }}$ \\ Quantum Nano-Electronics Research Center, Tokyo Institute of Technology, O-okayama, Meguro-ku, Tokyo \\ 152-8552, Japan
}

(Received 30 April 2004; accepted 10 August 2004)

\begin{abstract}
By using Kelvin probe force microscopy, we investigate the contact potential difference (CPD) of nanocrystalline silicon (nc-Si) dots with various sizes before and after the dots charging. Few electrons are injected into the nc-Si dots using the atomic force microscope tip. A remarkable change in the dot potential is observed under the normal ambient conditions. Since the change in the dot potential represents the charging energy of the nc-Si dots, the number of electrons stored in the individual dots can be estimated by comparing the calculated charging energy and the measured CPD change caused by charging. We demonstrate that charge quanta are indeed injected and directly detected by this method by analyzing the dot diameter dependence of the CPD change for each dot. (C) 2004 American Institute of Physics. [DOI: 10.1063/1.1804250]
\end{abstract}

The continuous evolution of modern electronics accelerates aggressive miniaturization of silicon device structures towards the decananometer regime. Plasma decomposition of $\mathrm{SiH}_{4}$ was proven to be a reliable method of forming nanocrystalline silicon (nc-Si) dots with the size down to a few nanometers, and it has attracted great interest from the standpoints of their unique properties of charge storage, ${ }^{1}$ cold electron emission, ${ }^{2}$ and photoluminescence. ${ }^{3}$

An efficient tool capable of resolving the nanometerscale local properties has been aspired for investigating the nc-Si-dot-based devices thoroughly. Although the conventional $I-V$ and $C-V$ characterization methods provide a vast amount of information, these methods lack the ability of discriminating structural and material properties on a nanometer scale. On the other hand, scanning probe microscopy (SPM) techniques, which are supposed to give the best approach to study nanostructures, facilitate the lateral and vertical resolutions of 0.1 and $0.01 \mathrm{~nm}$, respectively, by scanning a sharp tip across a sample surface. Furthermore, the Kelvin probe force microscopy (KFM) is preferred among the various types of SPM techniques when we need to observe the surface potential or work-function profiles. Since the first attempt was reported by Nonnemacher et al., ${ }^{5}$ the KFM has been used widely for investigating metal thin films, ${ }^{5,6}$ semiconductors $^{7,8}$ and nanoparticles. ${ }^{9}$

The operating principle of the KFM is setting off the electrostatic forces between the tip and the sample by applying a feedback potential. When two materials with different work functions are electrically connected, electrons move from the material with a low work function to that with a high one, since the work function reflects how strongly the electrons are bound in each material. This electron transfer gives rise to an electric field between the tip and the sample and, therefore, an electrostatic force in between. Since the atomic force microscope basically measures forces, a feedback circuit can provide a certain voltage in order to cancel this force, which is given by ${ }^{10}$

\footnotetext{
${ }^{\text {a)} E l e c t r o n i c ~ m a i l: ~ m a s a l e m @ d i a n a . p e . t i t e c h . a c . j p ~}$

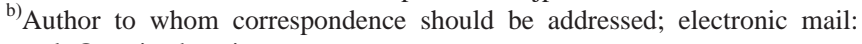
soda@pe.titech.ac.jp
}

$$
F=\frac{d C}{2 d z}\left[\Delta \Phi-\left(V_{d c}+V_{a c} \sin (\omega t)\right)\right]^{2},
$$

where $z$ is the tip-to-sample distance, $C$ the capacitance, $\Delta \Phi$ the contact potential difference (CPD) between the tip material and the sample material, and $V_{d c}$ and $V_{a c}$ are the dc and ac components of the applied voltage, respectively. Due to the applied ac voltage, the cantilever no longer vibrates mechanically, but electrically. The electrostatic force can be decomposed into three frequency-dependent (spectral) forces as follows:

$$
F=F_{d c}+F_{\omega}+F_{2 \omega},
$$

where three spectral force components at $\mathrm{dc}, \omega$, and $2 \omega$ are given as

$$
\begin{aligned}
& F_{d c}=\frac{1}{2} \frac{\partial C}{\partial z}\left[\left(\Delta \Phi-V_{d c}\right)^{2}+\frac{V_{a c}^{2}}{2}\right], \\
& F_{\omega}=\frac{\partial C}{\partial z} V_{a c} \sin (\omega t)\left(V_{d c}-\Delta \Phi\right), \\
& F_{2 \omega}=\frac{-1}{4} \frac{\partial C}{\partial z} V_{a c}^{2} \cos (2 \omega t) .
\end{aligned}
$$

The amplitude of cantilever vibration at a frequency $\omega$ will be directly proportional to the spectral component of the force at the frequency $\omega$ if $\omega$ equals the resonance frequency of the cantilever. Furthermore, if the $V_{d c}$ equals the CPD, $\Delta \Phi$, the force at $\omega$ would then be nullified. Recording the dc voltage with scanning the entire sample area therefore enables mapping the surface potential of the sample on the nanometer scale.

For preparing the dilute and isolated nc-Si dots suitable for our present study, a thin $\mathrm{SiO}_{2}$ layer was first grown using chemical oxidation of $\mathrm{H}_{2} \mathrm{SO}_{4} / \mathrm{H}_{2} \mathrm{O}_{2}$ on a $p$-type (100) $\mathrm{Si}$ substrate, and nc-Si dots about $2-8 \mathrm{~nm}$ in diameter were then deposited subsequently with plasma decomposition of $\mathrm{SiH}_{4}$ described in detail elsewhere. ${ }^{11} \mathrm{Ar}$ and $\mathrm{SiH}_{4}$ gases were ex- 


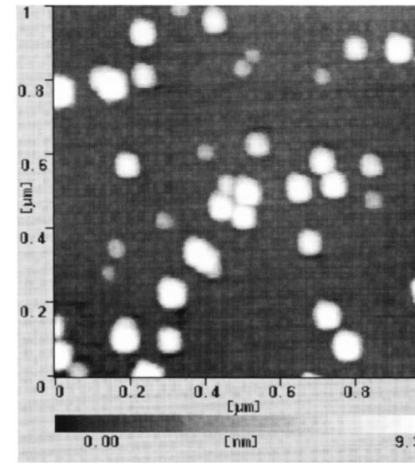

(a)



(b)

FIG. 1. Simultaneous (a) topographic and (b) potential KFM images for the nc-Si dots before charging.

cited by very high frequency (144 MHz) plasma, and the size and density of the nc-Si dots were controlled essentially via the duration and number of the $\mathrm{SiH}_{4}$ pulses.

Topographic and potential measurements were performed simultaneously under the normal ambient conditions using a commercially available AFM-based Kelvin probe force microscope (SPA400, Seiko Instruments Inc.) using a sharp (35-nm radius of curvature) rhodium-coated silicon cantilever having the resonance frequency of about $24 \mathrm{KHz}$ and the force constant of $1.8 \mathrm{~N} / \mathrm{m}$. The area under investigation is $1 \times 1 \mu \mathrm{m}$ and scanned at $0.87 \mathrm{~Hz}$, and with the 10 $\mathrm{V}$ peak-to-peak ac voltage.

Charge injection into the Si dots was carried out using the biased tip in the contact mode. This was done for the dots in a particular area selected in the previous scan in the noncontact mode. After positioning the conducting tip to the targeted point, the tip was biased at $-5 \mathrm{~V}$ and was then edged nearer to the surface and kept in contact for $30 \mathrm{~s}$. After that, the tip was retracted and grounded, and the subsequent noncontact mode KFM scan was performed; the change in the measured CPD of the dots upon charging indicates directly the charging energy added to each dot. It should particularly be noted that scanning the sample should not be done in the contact mode, as this would move the deposited dots away. Further details on the charging process using the AFM tip, as well as a theoretical model for estimating the charge transferred, can be found elsewhere. ${ }^{12}$

Figure 1 shows the simultaneously scanned (a) topography and (b) potential images. Note that the lateral dot dimensions are overestimated as the AFM image is given as a convolution of the sample and tip shapes. ${ }^{13}$ However, we may obtain the diameter of the individual dots much more precisely from the vertical topography data. The dot size was found to range from about $2-8 \mathrm{~nm}$. Due to atmospheric exposure, we assume that a thin $\mathrm{SiO}_{2}$ shell was grown naturally on the silicon dots. However, this oxidation process has been proven to be self-limiting in thickness rather than continuous. ${ }^{14}$ The potential image with high contrast is shown in Fig. 1(b), where the clear distinction between the Si dots and the substrate is seen. In addition, small and large dots are clearly distinguished in the potential distribution, and we found that the larger the dots the lower the CPDs. Despite a variety of studies done using the KFM in the past with the atomic scale lateral resolutions, ${ }^{8,15}$ our present work features a direct measurement of the size-dependent potential of the silicon quantum dots, although the obtained CPDs can be

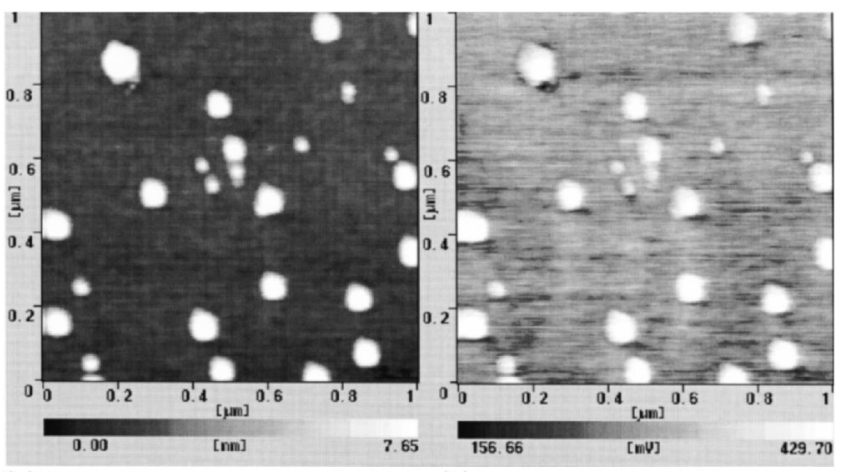

(a)

(b)

FIG. 2. Simultaneous (a) topographic and (b) potential KFM images for the nc-Si dots after charging.

affected by the existence of any adsorption layers on the surface. $^{10}$

Figures 2(a) and 2(b) show the images obtained after the charging process. As expected, the topographic height did not show any significant change after the charging, since the KFM measurement system cancels the electrostatic forces between the tip and the sample as explained earlier. Therefore, charging the sample has no effect on the sample topography obtained with the KFM. In that sense, the KFM is different from other noncontact or tapping mode measurements, in which the topographic images are indeed affected by all types of forces, and the charge transferred from tip to sample is estimated only in an indirect manner, in which the topographic height change (or apparent height) is analyzed. ${ }^{12,16}$ It is worth mentioning that the potential imaging is dramatically improved in this work compared with those reported in the past, ${ }^{17}$ where the distinction was unclear between the potential of dots and that of the substrate. This is attributable to the method that we used for charging the individual $\mathrm{Si}$ dots using the AFM tip without affecting the potential of the substrate overall. The time spent for switching from the contact mode to the noncontact mode, including the necessary adjustments, was about $5 \mathrm{~min}$. This was followed by another 5 min to complete the simultaneous topographic and potential scan.

The change in the dot spatial distribution after the charging is attributable to the repulsive forces between the charged dots. It is interesting to see that larger dots show the higher potential than that for smaller dots, while the substrate remains at almost the same potential of about $300 \mathrm{mV}$. This manifests that only the $\mathrm{Si}$ dots were charged and that the KFM facilitates to detect the size dependence of the charge confined in the nanostructures experimentally.

We can estimate the number of electrons injected into each dot by comparing the measured CPD change with the charging energy calculated for the Si dots, ${ }^{18}$

$$
E_{c}=\frac{(n e)^{2}}{C_{d o t}},
$$

where $E_{c}$ is the charging energy, $C_{d o t}=4 \pi \varepsilon d$ is the dot selfcapacitance calculated separately for individual dots, $\varepsilon$ is the dielectric constant of silicon, $d$ is the dot diameter, and $n$ is the number of injected electrons. Figure 3 shows the CPD changes (solid circles) caused by the charging process plotted as a function of the dot diameter. The dot diameter for the individual dots was estimated from the dot height data in AIP license or copyright; see http://apl.aip.org/apl/copyright.jsp 


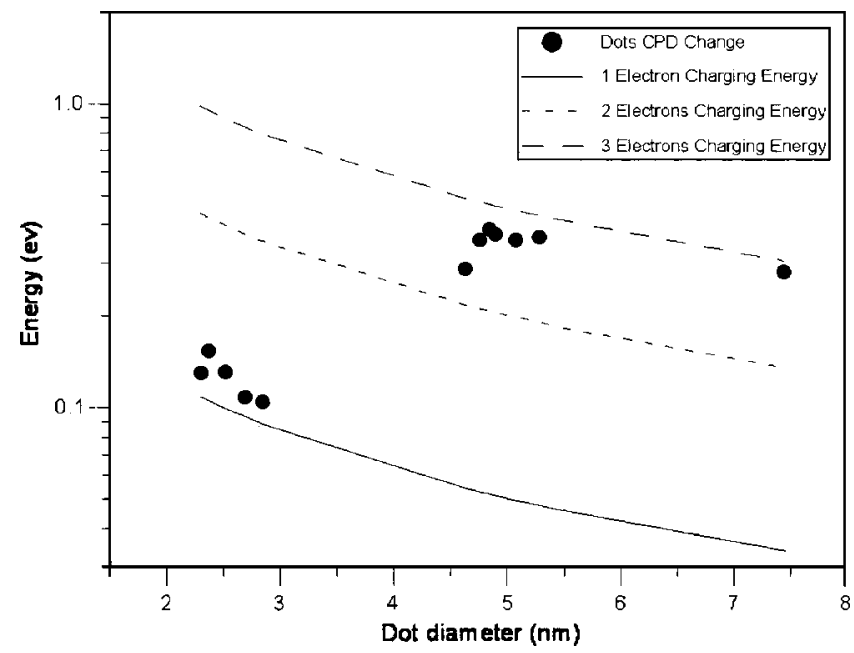

FIG. 3. The change in the CPD (contact potential difference) observed for the nc-Si dots with various values of dot diameter (solid circles) and the calculated charging energy as a function of the dot diameter with the number of extra electrons on the dot of one (a thin solid line), two (a dotted line), and three (a broken line).

Figs. 1(a) and 2(a) with their gray scale bars underneath. Figure 3 also shows the charging energy calculated by using Eq. (6) for the Si dots with one (a thin solid line), two (a dotted line), and three (a broken line) extra electrons per dot. These results indicate that only one electron is stored in the nc-Si dot with a diameter up to $2.8 \mathrm{~nm}$, and three electrons are stored in the dots with diameters in the range from 4.7 to $7.4 \mathrm{~nm}$. The dot with a diameter of $4.6 \mathrm{~nm}$ likely stores two electrons, although this is not very clear as we did not find the dots in the range of $2.8-4.7 \mathrm{~nm}$ in the present sample.

In conclusion, a simple, reliable, and precise method of charging nc-Si dots, as well as probing the charge injected into the dots, has been presented based on the simultaneous observation of the KFM topography and the CPD change of the dots. The number of injected electrons has been identified by comparing the measured CPD changes as a function of the dot diameter with the charging energy calculated for various numbers of electrons. This method may provide a powerful tool for investigating local electronic states and transport properties in the modern nanoelectronic devices.

M.A.S. is grateful to Dr. S. Banerjee for the fruitful discussions. The authors also are thankful to Dr. K. Usami for nc-Si dot deposition and to Dr. Y. Tsuchiya for the KFM set up. This work has been supported by Core Research for the Evolutional Science and Technology (CREST) Program of Japan Science and Technology (JST).

${ }^{1}$ B. J. Hinds, T. Yamanaka, and S. Oda, J. Appl. Phys. 90, 6402 (2001).

${ }^{2}$ K. Nishiguchi, X. Zhao, and S. Oda, J. Appl. Phys. 92, 2748 (2002).

${ }^{3}$ K. Arai and S. Oda, Phys. Status Solidi C 0, 1254 (2003).

${ }^{4}$ D. K. Schroder, Semiconductor Material and Device Characterization, 2nd ed. (Wiley, New York, 1998).

${ }^{5}$ M. Nonnenmacher, M. P. O'Boyel, and H. K. Wickramasinghe, Appl. Phys. Lett. 58, 2921 (1991).

${ }^{6}$ M. P. O’Boyel, T. T. Hwang, and H. K. Wickramasinghe, Appl. Phys. Lett. 74, 2641 (1999).

${ }^{7}$ F. Robin, H. Jacobs, O. Homan, A. Stemmer, and W. Bachtold, Appl. Phys. Lett. 76, 2907 (2000).

${ }^{8}$ S. Kitamura, K. Suzuki, M. Iwatsuki, and C. B. Mooney, Appl. Surf. Sci. 157, 222 (2000).

${ }^{9}$ S. Ono, M. Takeuchi, and T. Takahashi, Ultramicroscopy 91, 127 (2002).

${ }^{10}$ B. Bhushan and A. Goldade, Wear 244, 104 (2000).

${ }^{11}$ T. Ifuku, M. Otobe, A. Itoh, and S. Oda, Jpn. J. Appl. Phys., Part 1 36, 4031 (1997).

${ }^{12}$ S. Banerjee, M. A. Salem, and S. Oda, Appl. Phys. Lett. 83, 3788 (2003).

${ }^{13}$ T. Junno, K. Deppert, L. Montelius, and L. Samuelson, Appl. Phys. Lett. 66, 3627 (1995).

${ }^{14}$ K. Arai, J. Omachi, K. Nishiguchi, and S. Oda, Mater. Res. Soc. Symp. Proc. 664, A20.6.1 (2001).

${ }^{15}$ S. Kitamura and M. Iwatsuki, Appl. Phys. Lett. 72, 3154 (1998).

${ }^{16}$ E. A. Boer, L. D. Bell, M. L. Brongersma, and H. A. Atwater, J. Appl. Phys. 90, 2764 (2001).

${ }^{17}$ N. Shimizu, M. Ikeda, E. Yoshida, H. Murakami, S. Miyazaki, and M. Hirose, Jpn. J. Appl. Phys., Part 1 39, 2318 (2000).

${ }^{18}$ K. Likharev, Proc. IEEE 87, 606 (1999). 